\title{
Managing life-threatening haematoperitoneum in a non-pregnant patient secondary to bromadiolone self-poisoning
}

\author{
Authors: Patricia Cox and Dede Ofili-Yebovi
}

\begin{abstract}
Aims
This case report aims to highlight how to manage bromadiolone (superwarfarin) self-poisoning holistically across three different specialties, ruling out gynaecological causes of haematoperitoneum, correction of the resulting coagulation disorder, and psychiatric input.
\end{abstract}

\section{Methods}

A 21-year-old female was admitted under gynaecology, tachycardic and with heavy vaginal bleeding with bruising and no history of trauma. Haemoglobin was $29 \mathrm{~g} / \mathrm{L}$, which is unusual in this age group. Ultrasound showed a significant amount of free fluid in her pelvis, suggestive of blood, and she underwent laparoscopy which showed a $300 \mathrm{~mL}$ haematoperitoneum and no other pathology. She was given a stat dose of $\mathrm{GnRH}$ (gonadotropin-releasing hormone) analogue to prevent further ovulation, and a Mirena system was fitted, as well as a course of medroxyprogesterone.

Initial blood results showed prolonged PT (prothrombin time) and APTT (activated partial thromboplastin time), and normal fibrinogen levels. A sample was sent for quantification of factors, which showed low factor II, VII, IX and X. During the course of her stay, she admitted to taking a staggered overdose of bromadiolone over three episodes in a 3-4-week period, with a total of $250 \mathrm{~g}$ ingested. Liaison psychiatry were involved as she initially refused treatment, despite a risk of interventricular haemorrhage and was sectioned.

She was commenced on $20 \mathrm{mg}$ vitamin K orally per day over a period of 6 months to 1 year, with INR monitoring every 2-3 days. After 22 days of inpatient treatment, her INR range was satisfactory for her to be discharged.

\section{Results}

Bromadiolone is a second-generation 4-hydroxycoumarin derivative. It inhibits vitamin $\mathrm{K}(1)-2,3$ epoxide reductase and therefore synthesis of vitamin K1 clotting factors - factors II,
VII, IX and X - as demonstrated in the patient's blood results. It binds to the enzyme with a greater affinity than warfarin and can disrupt the cycle at more than one point, and accumulates in the liver. It also has a longer half-life than warfarin, due to high lipid solubility and enterohepatic circulation, hence the prolonged course of vitamin $\mathrm{K}$ and projected duration of treatment.

\section{Conclusions}

There are not a lot of published data on elimination kinetics and half-life of bromadiolone in humans, therefore treatment is guided by coagulation profile; however, there are no treatment guidelines. The coagulopathy can take a long time to reverse, especially in the above case as there was staggered self-poisoning. Clotting disorders are a rare cause of heavy vaginal bleeding in a young population, but one which must be considered.

\section{Conflict of interest statement}

There are no conflicts of interest. 